\title{
Parallelization of Reordering Algorithms for Bandwidth and Wavefront Reduction
}

\author{
Konstantinos I. Karantasis*, Andrew Lenharth ${ }^{\dagger}$, Donald Nguyen ${ }^{\ddagger}$, María J. Garzarán*, Keshav Pingali ${ }^{\dagger}$, \\ * Department of Computer Science, \\ University of Illinois at Urbana-Champaign \\ $\{$ kik, garzaran\}@illinois.edu
${ }^{\dagger}$ Institute for Computational Engineering and Sciences and
${ }^{\ddagger}$ Department of Computer Science, University of Texas at Austin
lenharth@ices.utexas.edu, \{ddn, pingali\}@cs.utexas.edu

\begin{abstract}
Many sparse matrix computations can be speeded up if the matrix is first reordered. Reordering was originally developed for direct methods but it has recently become popular for improving the cache locality of parallel iterative solvers since reordering the matrix to reduce bandwidth and wavefront can improve the locality of reference of sparse matrix-vector multiplication (SpMV), the key kernel in iterative solvers.

In this paper, we present the first parallel implementations of two widely used reordering algorithms: Reverse Cuthill-McKee (RCM) and Sloan. On 16 cores of the Stampede supercomputer, our parallel RCM is 5.56 times faster on the average than a state-of-the-art sequential implementation of RCM in the HSL library. Sloan is significantly more constrained than RCM, but our parallel implementation achieves a speedup of $2.88 \mathrm{X}$ on the average over sequential HSL-Sloan. Reordering the matrix using our parallel RCM and then performing $100 \mathrm{SpMV}$ iterations is twice as fast as using HSL-RCM and then performing the SpMV iterations; it is also 1.5 times faster than performing the SpMV iterations without reordering the matrix.
\end{abstract}

\section{INTRODUCTION}

Many sparse matrix computations can be speeded up by reordering the sparse matrix before performing the matrix computations. The classical examples are direct methods for solving sparse linear systems: in a sequential implementation, reordering the matrix before factorization can reduce fill, thereby reducing the space and time required to perform the factorization [14, 16]. In parallel implementations of direct methods, reordering can reduce the length of the critical path through the program, thereby improving parallel performance [11]. Reordering can also reduce the amount of storage required to represent the matrix when certain formats like banded and skyline representations are used [16].

In most applications, computing the optimal reordering is NP-complete; for example, Papadimitriou showed that reordering to minimize bandwidth is NP-complete [38]. Therefore, heuristic reordering algorithms are used in practice, such as minimum-degree, Cuthill-McKee (CM), Reverse CuthillMcKee (RCM), Sloan, and nested dissection orderings [12, 16, [26, [33, 43]. Some of these reordering methods are described in more detail in Section $\mathbf{m}$.
More recently, reordering has become popular even in the context of iterative sparse solvers where problems like minimizing fill do not arise. The key computation in an iterative sparse solver is sparse matrix-vector multiplication (SpMV) (say $y=A x$ ). If the matrix is stored in compressed rowstorage (CRS) and the SpMV computation is performed by rows, the accesses to $y$ and $A$ enjoy excellent locality, but the accesses to $x$ may not. One way to improve the locality of accesses to the elements of $x$ is to reorder the sparse matrix A using a bandwidth-reducing ordering (RCM is popular). In this context, the purpose of a reordering technique like RCM is to promote cache reuse while a single core (or a set of cores cooperatively sharing some level of cache) perform the computation.

When a large cluster is used to perform the SpMV computation, the matrix is either assembled in a distributed manner [177] or a partitioner like ParMetis is used to partition the matrix among the hosts, and each partition is then reordered using a technique like RCM to promote locality. This use of reordering was first popularized in the Gordon Bell prize-winning paper at SC1999 by Gropp et al. [2, 20]. Local reordering with algorithms like RCM are now used widely in parallel iterative solvers.

In all these applications, reordering is performed sequentially even if the sparse matrix computation is performed in parallel. In the traditional use-case of reordering for sparse direct methods, this is reasonable because reordering takes a very small fraction of the overall execution time, which is dominated by the cost of numerical factorization [16]. However, when reordering is used to improve cache locality for sparse iterative methods, reordering time can be roughly the same order of magnitude as the SpMV computation. Therefore, it is useful to parallelize the reordering algorithms too.

Parallelizing reordering algorithms like RCM or Sloan is very challenging because, although there is parallelism in these algorithms, the parallelism does not fall into the simple dataparallelism pattern that is supported by existing frameworks such as OpenMP and MPI. In fact, these algorithms belong to 
a complex class of algorithms known as irregular algorithms in the compiler literature [40]. These algorithms exhibit a complex pattern of parallelism known as amorphous dataparallelism, which must be found and exploited at runtime because dependences between parallel computations are functions of runtime data values. These complexities have deterred previous researchers from attempting to parallelize reordering algorithms like RCM.

In this paper, we describe the first ever parallelizations of two popular reordering algorithms, Reverse Cuthill-McKee (RCM) [112, [16] and Sloan [43]. Both are based on performing traversals over the graph representation of a sparse matrix. We use the Galois system to reduce the programming burden [15]. Since reordering strategies like RCM and Sloan are heuristics, we allow our parallel implementation of a reordering algorithm to produce a reordering that may differ slightly from the one produced by the sequential algorithm, if this improves parallel performance. Since this may impact the SpMV performance, this flexibility has to be studied experimentally to determine the impact of parallel reordering on the overall execution time.

Because we are interested only in reordering matrices within a single host to improve the cache performance of SpMV, we restrict ourselves to single host studies. Our parallel RCM implementation obtains similar quality results as those from a state-of-the-art sequential implementation in the HSL library [23] and achieves speedups ranging between 3.18X to $8.92 \mathrm{X}$ on 16 cores with an average improvement of $5.56 \mathrm{X}$ across a suite of sparse matrices. Reordering the matrix using our parallel RCM and then performing $100 \mathrm{SpMV}$ iterations is twice as fast as using HSL-RCM and then performing the SpMV iterations; it is also 1.5 times faster than performing the SpMV iterations without reordering the matrix.

The rest of this paper is organized as follows. Section II presents the background on the reordering mechanisms and Galois, the programming model that we use to implement our parallel algorithms. Section $\mathrm{II}$ describes the parallel implementations of BFS, RCM, and Sloan. Section DV presents the experimental results. Section $\nabla$ discusses the related work, and Section VI concludes.

\section{BACKGROUND}

The bandwidth of a matrix is the maximum row width, where the width of row $i$ is defined as the difference between the column index of the first and the last non-zero elements in row $i$. If the matrix is symmetric, then the bandwidth is the semi-bandwidth of the matrix, which is the maximum distance from the diagonal. In a matrix with bandwidth $k$, all the nonzeros are at most $k$ entries from the diagonal.

Reordering mechanisms can be applied to reduce the bandwidth of a matrix. While few matrices are banded by default, in many cases the bandwidth of a matrix can be reduced by permuting or renumbering its rows and columns. The original structure of the matrix can be recovered by inverting the permutation.

Reducing bandwidth is usually applied as a preprocessing step for sparse matrix-vector multiplication [39] and some preconditioners for iterative methods [7]. Additionally, gathering or clustering non-zeros around the diagonal can be useful in graph visualization [24, 36]. However, for direct methods such as Cholesky or LU factorization, bandwidth reduction may actually hurt performance since it can increase fill. Some reordering strategies for direct methods try to reduce the wavefront of the matrix, where the wavefront for an index $i$ of a matrix is defined as the set of rows that have non-zeros in the submatrix consisting of the first $i$ columns of the matrix and rows $i$ to $N[28]$.

In this paper, we consider parallelizations of two popular reordering algorithms, Reverse Cuthill-McKee (RCM) [12], which attempts to reduce the bandwidth of symmetric matrices, and Sloan [43, 44], which tries to minimize the wavefront. Next, we describe Galois, the library and runtime sytem that we use to implement our algorithms (Section $\mathbb{\text { I-A }}$ ). Then, we describe breadth-first search (BFS) (Section II-B), an important subroutine to both algorithms. In the subsequent sections, we describe the sequential versions of RCM (Section II-C) and Sloan (Section ㅍ-D).

\section{A. Galois Programming Model}

The Galois system is a library and runtime system to facilitate parallelization of irregular algorithms, in particular, graph algorithms [15, 27]. The Galois system adds two concepts to a traditional sequential programming mode: ordered and unordered set iterators. Set iterators are indicated by the foreach construct in our figures. Set iterators in Galois differ from conventional set iterators in that new elements can be added to a set while it is being iterated over. Apart from that, an unordered set iterator behaves like an iterator in traditional programming languages except that there is no specific order that elements of a set are traversed and that iterations can add new elements to the set during traversal. The new elements will be processed before the loop finishes.

An unordered set iteration can be parallelized by processing multiple iterations at once and checking if concurrent iterations access the same data. Conflicting iterations can be rolled back and tried again later. This process will generate some serialization of iterations, which is a correct execution according to the definition of the unordered set iterator. There are various optimizations that the Galois system applies to reduce the overhead of speculative parallelization, and in practice, it is able to achieve good speedup for many irregular algorithms [40].

When iterations of an unordered loop statically have no dependences on each other nor are any new elements added, the loop is equivalent to parallel DOALL.

An ordered set iterator is like an unordered set iterator with an additional restriction: the serialization of iterations must be consistent with a user-defined ordering relation on iterations. An ordered set iterator is a generalization of thread-level speculation or DOACROSS loops [37]. In those approaches, the ordering relation is the sequential iteration order of a loop. An ordered set iterator, on the other hand, allows for user-defined ordering. Supporting the general case can 


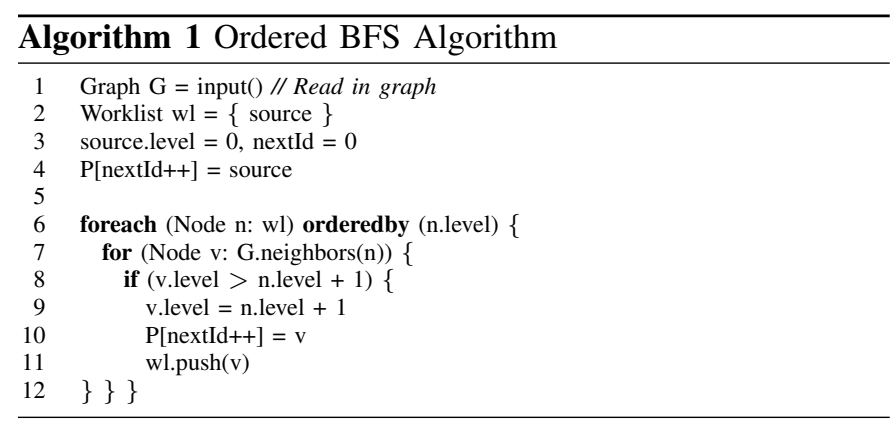

have significant runtime and synchronization overhead. One important optimization is to break down ordered set iterators into sequences of unordered set iterators when possible. In the following sections, we introduce algorithms using ordered set iterators for simplicity but our parallelizations in Section سW reformulate the algorithms into unordered forms for performance.

\section{B. Breadth-First Search}

Breadth-first search (BFS) is a common primitive in reordering algorithms, where it is used either as a subroutine or as an algorithmic skeleton for more complicated graph traversals.

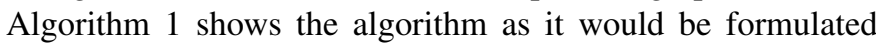
in the Galois system using an ordered set iterator. We call this the ordered BFS algorithm. The body of the outer loop is the operator. It is applied to nodes in a worklist. Initially, the worklist contains a single element, the source node from which to start the BFS. Each node has a level value that indicates its distance from the source. Level values are initialized with a value that is greater than any other level value. Worklist elements are processed in ascending level order. The $B F S$ parent of a node $n$ is the neighbor of $n$ that first updated the level of $n$. The source node of the BFS has no parent.

BFS itself can be used as a reordering algorithm, in which case, the permutation it generates is simply the order that nodes are visited. In the algorithm, this is recorded in the array $P$.

The ordering relation in Algorithm [ has two important properties: (i) level values are generated and processed in monotonically increasing order, and (ii) a nodes with level $l$ adds nodes to the worklist with level $l+1$ only. This order produces a work-efficient BFS implementation but constrains parallelism because, although all the nodes at level $l$ can be processed simultaneously, no node at level $l+1$ can be processed until all the nodes at level $l$ are done. Barriers are often used to synchronize threads between levels.

BFS can also be formulated as a fixed-point algorithm, in which operations can be done in any order corresponding to a chaotic relaxation scheme [9], rather than the order specified on line 5. This unordered parallelization of BFS can be derived by noticing that the BFS level of a node is a local minimum in the graph, i.e., the level of a node (except for the root) is one more than the minimum level of its neighbors. The body of the loop, sometimes called a node relaxation in the literature, will only lower the distance on a node as new paths

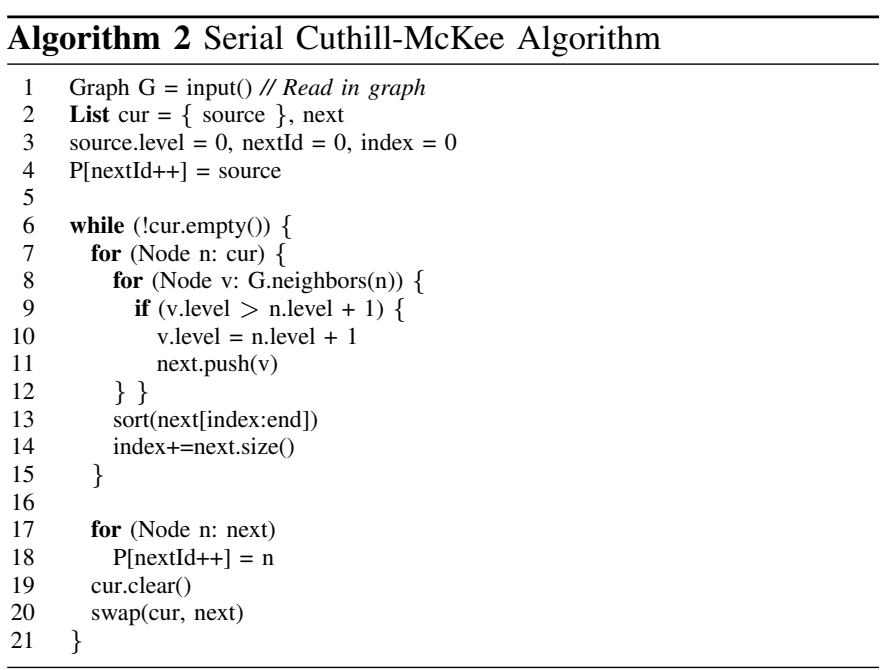

are discovered from the source to the node. Applying node relaxations eventually reaches a fixed-point with correct BFS levels regardless of the order in which relaxations are applied. We call this implementation unordered BFS, and the pseudocode for unordered BFS is the same as the one in Algorithm W, but without the orderedby clause in line 5.

A difference between the ordered algorithm and the unordered one is that with unordered BFS a node can be added to the $w l$ set (line 10) several times, and thus the level of a node may be updated multiple times, but correct final level values can only be determined after all the iterations have completed. For that reason, with unordered BFS it is not possible to incrementally produce the permutation array (line 9) in Algorithm W. If the permutation array is needed, a separate pass must be performed with the final level values.

Unordered BFS is used as a common building block in our parallel reordering algorithms because it scales better than the ordered algorithm, which must ensure that nodes at level $l+1$ are processed only after all nodes of level $l$. On the other hand, the unordered algorithm can lead to processing a node more than once. The extra iterations can be reduced by careful scheduling, which attempts to follow the scheduling of the ordered algorithm, but unlike the ordered algorithm, allows for some deviation in exchange for reduced synchronization.

\section{Cuthill-McKee}

The Cuthill-McKee (CM) [12] algorithm uses a refinement of BFS to produce a permutation. In addition to ordering nodes by level, nodes with the same level are visited in order of their BFS parent's placement in the previous level (choosing the earliest parent in case multiple neighbors are in the previous level) and in ascending degree order (or descending degree order for Reverse Cuthill-McKee) for nodes with the same earliest BFS parent. Reverse Cuthill-McKee (RCM) uses the opposite ordering relation so nodes are visited in descending degree order. Since it has been proven that RCM produces permutations of better or equal quality to the original Cuthill-McKee [34], we will mainly be concerned with Reverse Cuthill-McKee (RCM) in this paper, although 


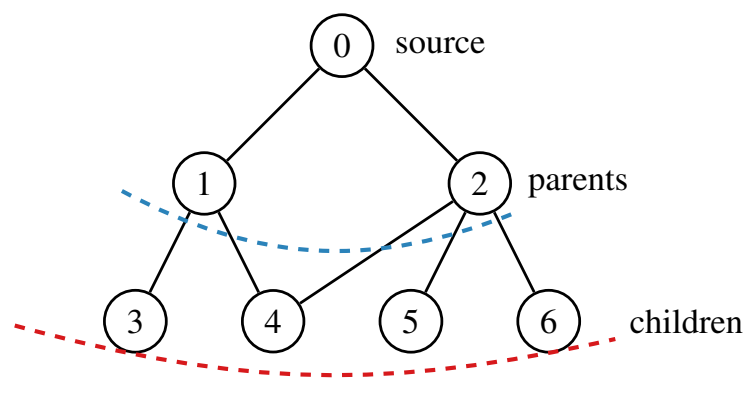

Fig. 1. Example of Cuthill-McKee

similar techniques can be applied for Cuthill-McKee.

Since RCM only adds ordering constraints to BFS, the traversals and permutations generated can be viewed as choosing a specific traversal out of the many possible BFS traversals. Algorithm $\square$ gives a serial implementation. Lines 5 to 11 implement a serial BFS. However, RCM requires sorting the nodes by degree (line 12) and inserts nodes in the permutation array as well as in the worklist for the next level in that order (lines 16 to 17 ).

There are some issues parallelizing RCM compared to the

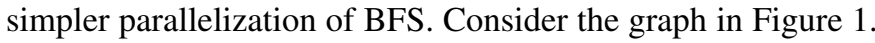
After executing the first iteration of the serial RCM algorithm, the worklist contains nodes 1 and 2. In BFS, these nodes can be processed in any order, as long as none of the successors of nodes 1 and 2, namely nodes 3, 4, 5 and 6, are processed before any of their parents (i.e., nodes 1 and 2). However, in RCM, node 1 should be processed before node 2 because it has smaller degree, and since processing a node also adds nodes to be processed in the next level, the children of node 1 (nodes 3 and 4) should be processed before the children of node 2 (nodes 5 and 6 ) in the next level.

\section{Sloan algorithm}

The Sloan algorithm [43, 44] is a reordering algorithm that attempts to reduce the wavefront of a graph. It is based on a prioritized graph traversal; nodes are visited in descending priority order. For a given start and end node $e$, the priority of a node $i$ is the weighted sum: $P(i)=-W_{1} * \operatorname{incr}(i)+$ $W_{2} *$ dist $(i, e)$ where incr $(i)$ is the increase of the size of the wavefront if node $i$ were chosen next and $\operatorname{dist}(i, e)$ is the BFS distance from node $i$ to the end node. The value of dist $(i, e)$ is fixed for a given graph and nodes $i$ and $e$, but the value of $\operatorname{incr}(i)$ changes dynamically as the permutation is constructed.

Algorithm B gives a sketch of the Sloan algorithm and shows how incr $(i)$ is updated. At each step, a node in the graph can be in one of these four states: (i) numbered; (ii) active, a non-numbered node that is a neighbor of a numbered node; (iii) preactive, a non-numbered and non-active node that is a neighbor of an active node; and (iv) inactive, all other nodes. Initially the source node is preactive and all other nodes are inactive. The algorithm iterates through all the nodes in the graph and at each step it chooses among the active or preactive

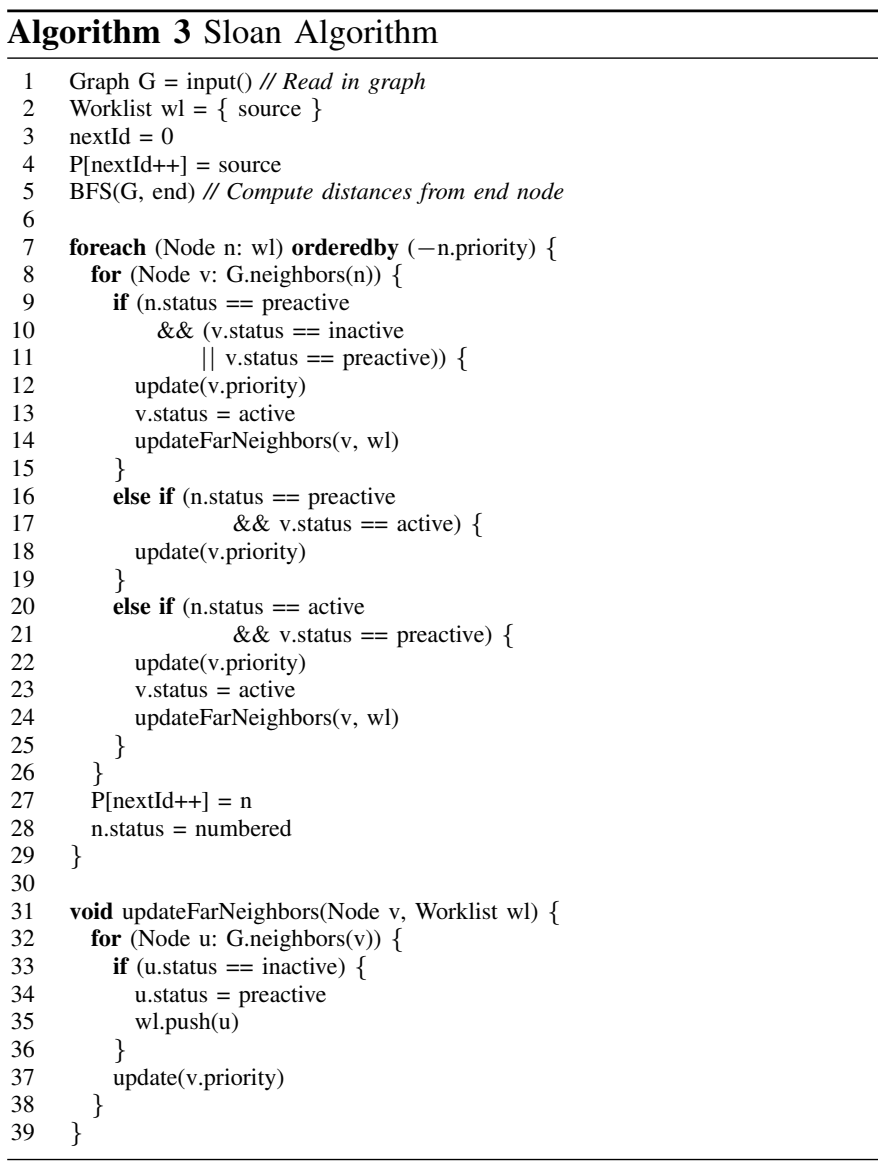

nodes the one that maximizes the priority. New priorities are assigned to the neighbors and the neighbors of the neighbors of the node selected. Details about how to compute the new priority of a node are not discussed as they are not important for the parallelization of the algorithm, but they can be found in [43, 44].

\section{E. Choosing Source Nodes}

Empirical data shows that the quality of reordering algorithms is highly influenced by the nodes chosen as the source for BFS and RCM or the source and end for Sloan [14, 41, 43]. For this work, we use the algorithm described by Kumfert [29] that computes a pair of nodes that lie on the pseudo-diameter, called pseudo-peripheral nodes. The diameter of a graph is the maximum distance between any two nodes. The pseudodiameter is a lower bound on the diameter that is simpler to compute. Pseudo-peripheral nodes, therefore, are a pair of nodes that are "far away" from each other in the graph. For BFS and RCM reordering, we pick one element from the pair to be the source.

The main computation in computing the pseudo-diameter is performing multiple breadth-first searches, which can be parallelized using the ordered or unordered algorithm. In the rest of the paper, we refer to the procedure for selecting source nodes as the pseudo-diameter computation. 


\section{PARAllel ReORdering}

In this Section we describe parallel implementations of BFS reordering (Section III-A), Reverse Cuthill-McKee (Section [II-B), and Sloan (Section WI-C).

\section{A. $B F S$}

A simple reordering algorithm is to use the traversal order generated by BFS. As mentioned in Section [I-B, a possible parallel implementation is the ordered BFS algorithm (see Algorithm $\mathbb{W}$ ). Another implementation is to use the unordered BFS algorithm described in Section II-B]. The unordered algorithm has the benefit of not requiring serialization of iterations like Algorithm M, but it cannot directly be used as a reordering algorithm because it does not generate a permutation directly. Instead, it only generates the final BFS levels for each node.

In this section, we describe how to take the output of the unordered BFS algorithm and generate, in parallel, a permutation by choosing one that is consistent with the levels generated by the unordered BFS.

Algorithm 10 gives the general structure. There are four major steps:

1) Compute the levels for each node using the unordered BFS algorithm.

2) Count the number of nodes at each level. In our algorithm, we compute the histograms locally for each thread and then sum them together.

3) Compute the prefix sum of the final histogram. The prefix sum gives the beginning offset in the final permutation array for nodes in any given level. Calculating prefix sums in parallel is well-known.

4) Finally, place nodes in the permutation array. This is done by dividing the nodes between threads and setting each node's position in the permutation to the next free position for the node's level. The next free position is computed from the current value in the prefix sum array for the level, and it is reserved by atomically incrementing that value. The value of $\operatorname{sums}(l)$ is the next location in the permutation array to be used by a node of level $l$.

This algorithm is non-deterministic. It will always produce a permutation that is consistent with a BFS ordering of nodes, but the specific serialization will depend on the number of threads, the layout of the graph in memory (e.g. the iteration order over the graph), and the interleaving of the atomic increments.

\section{B. Reverse Cuthill-McKee}

The RCM algorithm, like BFS, depends on the construction of a level structure to produce a permutation, but it is more challenging to parallelize because it places additional restrictions on valid permutations. We present two parallelizations of RCM, which differ on how to surmount this challenge. The first algorithm uses an incremental approach; at each major step of the algorithm, the permutation for the nodes seen so far is calculated and does not change in subsequent steps. We call this the leveled RCM algorithm. The second algorithm uses an

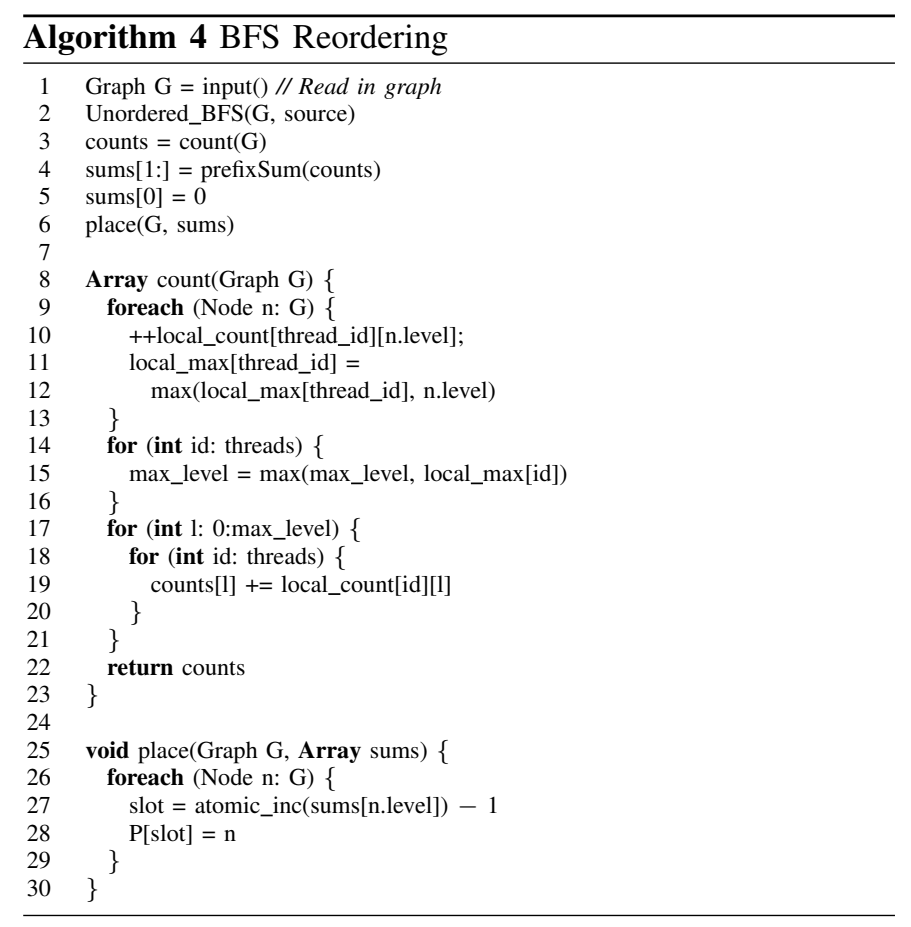

a posteriori approach that builds an RCM-valid permutation right after a complete level structure is computed. We call this the unordered RCM algorithm.

1) Leveled Reverse Cuthill-McKee: The leveled algorithm follows the general structure of the serial RCM Algorithm $\square$. It proceeds in iterations, where each iteration expands the frontier of the graph to the next level. The leveled algorithm parallelizes the processing of nodes per level. Each iteration consists of a sequence of steps, where each step executes in parallel with an implicit barrier between steps, and where each step executes as an unordered set iterator. When an iteration completes, nodes in a level have been conclusively added to the RCM permutation array. The algorithm is shown in Algorithm [1. The major steps are as follows.

1) The expansion step takes a list of nodes to expand in parallel. These parent nodes examine their neighbors to find their child nodes. As in BFS, when a neighbor is accessed for the first time, its distance from the source is recorded. Such node is considered a child node in this iteration. In addition, the appropriate parent is recorded for each child. Out of all the possible parents, the parent recorded is the one closer to the source node in the permutation array.

2) The reduction step computes the number of children for each parent.

3) A prefix sum step computes for each child an index in the permutation array according to each child's parent.

4) The placement step performs the actual placement of children in the permutation array. Every child node is placed at the designated range of indices determined for each parent during the previous step. The sequence of these ranges respects the RCM ordering of parents. The placement of children ends by sorting the children of 


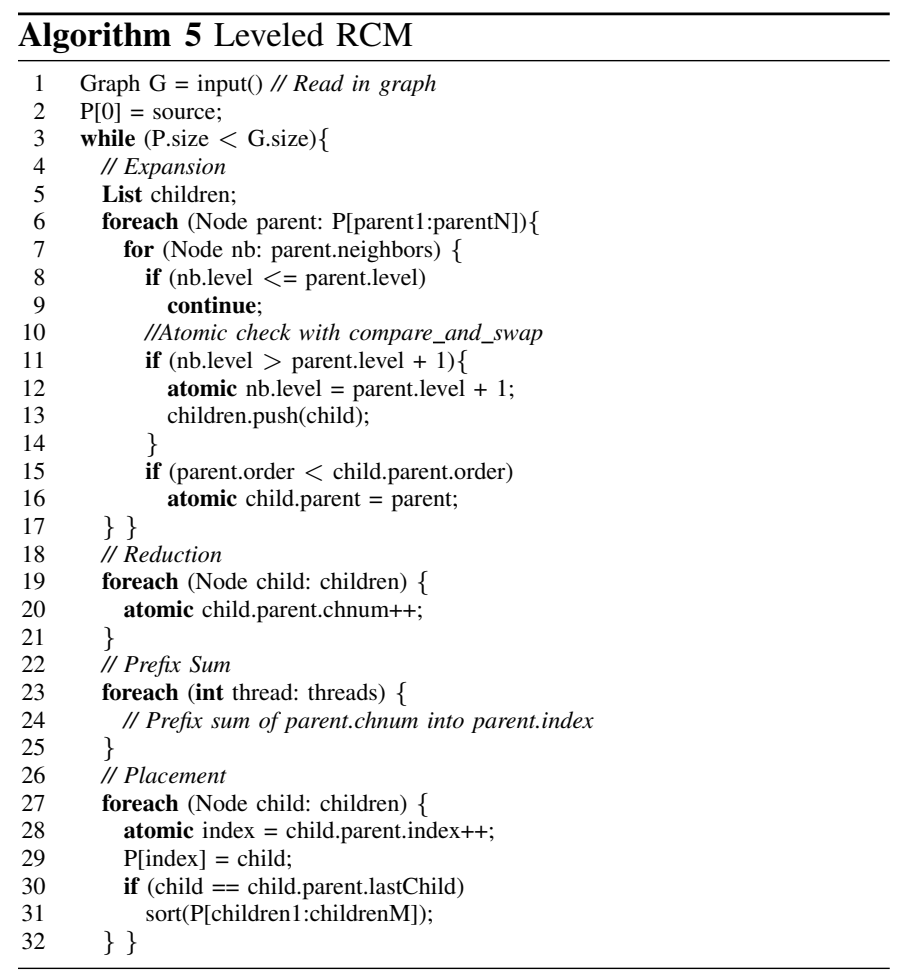

each parent in the permutation array in ascending degree.

2) Unordered Reverse Cuthill-McKee: We can also parallelize the RCM algorithm based on the parallel unordered BFS algorithm presented in Section II-B]. Algorithm 6 shows the implementation. It resembles the parallel BFS reordering algorithm, but with a more strict placement phase. Its first three major steps are the same. It computes BFS levels, counts the number of nodes at each level, and computes the prefix sum of levels.

Where this algorithm differs from parallel BFS placement is in the placement of nodes in the final permutation array. RCM specifies a specific placement order, whereas BFS allows more relaxed orders. The place function for unordered RCM thus has to enforce more ordering constraints than the one for BFS does. Given a correct ordering of one level, one can process nodes in that order to produce a correct ordering for the next level. We take advantage of this fact to pipeline the placement phase. We assign a single thread to each level, and threads communicate to the next level through a single-producer, single-consumer queue. Thus, a thread receives nodes in the correct order for its level and produces nodes in the correct order for the next level.

RCM orders nodes in two ways. First, nodes in a level are sorted according to the ordering of their parent in the previous level (using the least parent in case of multiple parents). This order is trivially respected since each node is processed in the order it was produced by the previous level, which is in order of its parent. The second ordering RCM uses is that nodes with the same parent are ordered by degree. This is ensured by having the thread, when processing a node, sort the child nodes by degree before forwarding them to the next level. As nodes are forwarded, they may also be placed in the

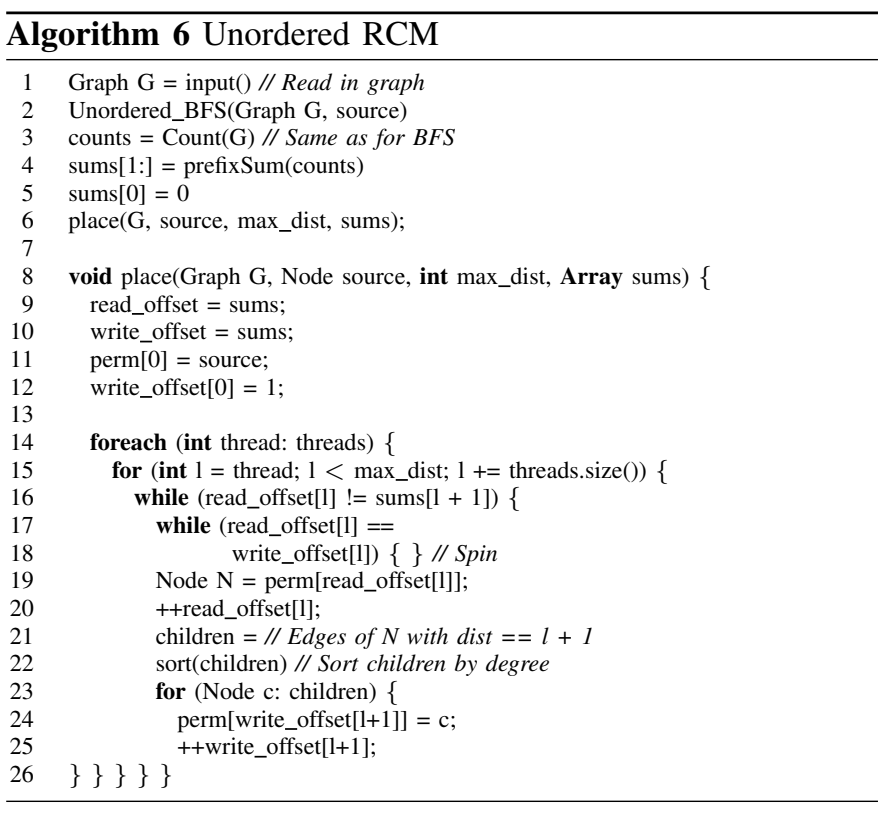

permutation array.

The permutation array does double duty both as the final ordering of nodes and as a series of single-producer, singleconsumer queues. Each logical queue is implemented as a read and write pointer into the portion of the permutation array corresponding to a given level. The portion of the array for each level is computed using the prefix sum over the number of nodes in each level of the BFS tree. Since the processing order is identical to the permutation order for a level, we can combine these two structures.

The placement phase is parallelized by pipelining. A thread at level $l$ writes out children in a RCM order for a thread at level $l+1$ to consume. Parallelization is achieved because each level can be populated as soon as nodes are produced by the previous level.

\section{Sloan}

The most natural parallelization of Sloan's algorithm is to process multiple iterations of the outermost loop in parallel. There are two main challenges to this dimension of parallelization. First, the outer loop relies on a priority queue to determine the next active node to examine. Second, for each active node, a significant portion of the graph must be manipulated, and it is likely that two successive active nodes will have overlapping neighborhoods.

Priority queues are difficult to make scalable and concurrent because threads contend on accesses to the head of the queue. When each iteration must select the next highest priority element to process, the priority queue becomes a scalability bottleneck. Two ways to increase the scalability of priority queue-based iterative algorithms are to find a more scalable data structure that achieves the same effect as the priority queue or to relax the requirement that items be retrieved in strict priority order.

One example of finding a more scalable data structure is in BFS. In principle, the worklist in serial BFS used to identify 
the next node to expand can be implemented with a priority queue that orders nodes in ascending distance. But, since the priority of newly enqueued nodes is always lower than that of all the current nodes in the queue, one can implement the priority order using a simple FIFO queue. In the parallelization of BFS, one can take this line of reasoning one step further. FIFO order is not strictly necessary to ensure the correct order of processing nodes. As long as all the nodes with distance $l$ are processed before any of the nodes that they will enqueue (at distance $l+1$ ), the algorithm will still be correct. In particular, the nodes at distance $l$ can be processed in any order not just FIFO order.

A hurdle to finding a more scalable data structure for Sloan's algorithm is that, due to the priority structure of the algorithm, if there are two nodes $a, b$ that should be processed in that order given the current state of the algorithm, processing node $a$ may introduce a node $c$ that should be processed before $b$. To see why this is the case, recall that the updatePriority function increases the priority of a node and its neighbors and the limit of the priority of a node depends on the connectivity of the graph and the weight parameters. Thus, without this runtime knowledge, it is not possible to limit the occurrences of these "intervening" nodes at compile time.

There are two main avenues of parallelization left: the first one is a just-in-time analysis which constructs a parallel schedule once the graph and weight parameters are known; the second one is an optimistic runtime parallelization which assumes that for some sequence of nodes that there will not be any intervening nodes and then verifies whether that is the case after executing the sequence in parallel. Both these approaches have significant overhead compared to the simplicity of the original sequential algorithm.

Our solution is to instead relax the strict priority order. Since Sloan's algorithm is already heuristic, perturbing the execution order is not catastrophic. In Section $\mathbb{Z}$, we quantify the change in results when allowing deviations from the sequential algorithm. The Galois system provides an approximate priority queue which attempts to return elements in priority order but may admit some number of priority inversions when a node is retrieved out of order [3]]. Internally, the approximate priority queue is implemented by thread-local maps that associate priority levels with concurrent bag data structures that store all the elements with that priority. There is a unique bag for each priority. Threads ensure this by using a versioned global log to synchronize new priority-bag entries. Periodically, threads read the log to update their thread-local maps and to find the highest priority non-empty bag.

The other main challenge to parallelization of Sloan's algorithm is that two successive active nodes will likely write to overlapping regions of the graph. Each activity updates the active node's neighbors and its neighbors' neighbors. Given that we would like to scale to tens of threads and the diameter of many of our input matrices is quite small (i.e., $<1000$ ), the probability that at least two concurrent activities will update the same node is quite high. If we want the parallelization to be serializable, each one of those conflicting activities must be

\begin{tabular}{llrr}
\hline Input & Type & \multicolumn{1}{c}{$\mathrm{N}$} & \multicolumn{1}{c}{ NNZ } \\
\hline inline_1 & SPD & 503,712 & $36,816,170$ \\
audikw_1 & SPD & 943,695 & $77,651,847$ \\
dielFilterV3real & SYM & $1,102,824$ & $89,306,020$ \\
G3_circuit & SPD & $1,585,478$ & $7,660,826$ \\
M6 & SYM & $3,501,776$ & $21,003,872$ \\
333SP & SYM & $3,712,815$ & $22,217,266$ \\
NLR & SYM & $4,163,763$ & $24,975,952$ \\
hugetric-00020 & SYM & $7,122,792$ & $21,361,554$ \\
delaunay_n24 & SYM & $16,777,216$ & $100,663,202$ \\
road_usa & SYM & $23,947,347$ & $57,708,624$ \\
\hline \multicolumn{4}{c}{ TABLE I }
\end{tabular}

TYPE, DIMENSION (N) AND NUMBER OF NON-ZEROS (NNZ) OF THE SPARSE MATRICES SELECTED FROM THE UNIVERSITY OF FLORIDA SParse Matrix Collection. SPD: Symmetric Positive Definite, $S Y M$ : SYMMETRIC.

serialized with respect to the others. Since we are already using an approximate priority order, our solution to this problem is to not guarantee serializability of activities. Instead, we make atomic each individual update to a node using compareand-swap instructions, but we make no effort to ensure the atomicity of a whole iteration.

\section{EXPERIMENTAL RESULTS}

In this section, we describe the evaluation of our leveled and unordered RCM and Sloan algorithms with well-known third-party implementations from the HSL mathematical software library [23]. Section $\mathbb{D}-A$ describes our evaluation methodology. Section $\mathbb{E - B}$ describes the reordering quality of our algorithms with respect to reductions in bandwidth and wavefront metrics. Section $\mathbb{D V}-\mathrm{C}$ evaluates both execution time and reordering quality across a suite of sparse matrices. In addition, we use sparse matrix-vector product (SpMV) to assess the impact of our parallel reordering schemes on the overall execution time of potential applications.

To evaluate our algorithms, we selected a set of ten symmetric matrices, each with more than seven million non-zeros, from the University of Florida Sparse Matrix Collection [113]. The selected matrices are shown in Table $\mathbb{l}$. All matrices are square and have only one strongly connected component. The matrices are also symmetric. Beyond these requirements, we filtered selections to maximize dataset diversity (no two matrices are from the same field) and excluded already banded matrices. In Table $\mathbb{I}$ as well as in the following tables input matrices are sorted in descending percentage of density ${ }^{\mathrm{m}}$.

\section{A. Methodology}

We evaluate four parallel reordering algorithms: BFS in Algorithm 1 using the unordered BFS described in Section [I-B]; two RCM algorithms, the leveled RCM in Section [I-B] and the unordered RCM in Section $\mathbb{I I - B} 2$; and Sloan in Section III-C. These algorithms were implemented in C++ using the Galois library v2.1.9 [15] and were compiled using GNU g++, version 4.7.1.

We compare our results with those obtained by HSL [23], a state-of-the-art mathematical software library that contains a

\footnotetext{
${ }^{1}$ We measure density as the percentage of non-zeros over the total number of matrix elements.
} 


\begin{tabular}{lrrrrr}
\hline & & HSL & & Galois \\
\cline { 5 - 6 } Input & Initial & RCM & \multicolumn{1}{c}{ BFS } & RCM \\
\hline inline_1 & 502403 & 6009 & & $11405(1 \%)$ & $\mathbf{6 0 0 2}$ \\
audikw_1 & 925946 & 35949 & & $64723(1 \%)$ & $\mathbf{3 4 8 7 2}$ \\
dielFilterV3real & 1036475 & 23772 & & $46681(5 \%)$ & $\mathbf{2 3 7 2 8}$ \\
G3_circuit & 947128 & $\mathbf{5 0 6 9}$ & & $9170(10 \%)$ & 5078 \\
M6 & 3492131 & 4170 & & $8279(0 \%)$ & $\mathbf{4 1 6 9}$ \\
333SP & 3681176 & $\mathbf{1 7 9 4 1}$ & & $52051(0 \%)$ & 18376 \\
NLR & 4163523 & 7829 & & $19696(0 \%)$ & $\mathbf{7 8 2 3}$ \\
hugetric-00020 & 7122285 & 3593 & & $8435(0 \%)$ & $\mathbf{3 4 9 0}$ \\
delaunay_n24 & 16769102 & $\mathbf{2 3 4 9 4}$ & & $56617(1 \%)$ & 28659 \\
road_usa & 22895468 & $\mathbf{6 8 2 8}$ & & $12873(4 \%)$ & 6830 \\
\hline \multicolumn{3}{c}{ TABLE II } & &
\end{tabular}

BANDWIDTH METRICS FOR THE DIFFERENT ALGORITHMS. BEST RESULTS APPEAR IN BOLD.

\begin{tabular}{|c|c|c|c|}
\hline & & HSL & Galois \\
\hline Input & Initial & Sloan & Sloan \\
\hline inline_1 & 130603 & 1776 & $1754(0 \%)$ \\
\hline audikw_1 & 477149 & 8570 & $8749(1 \%)$ \\
\hline dielFilterV3real & 598949 & 4182 & $4339(2 \%)$ \\
\hline G3_circuit & 85516 & 1468 & $1478(1 \%)$ \\
\hline M6̄ & 1236350 & 2391 & $2391(0 \%)$ \\
\hline 333SP & 600987 & 6941 & $\mathbf{6 5 0 3}(10 \%)$ \\
\hline NLR & 1444100 & 4359 & $4331(1 \%)$ \\
\hline hugetric- 00020 & 1283170 & 2101 & $2171(0 \%)$ \\
\hline delaunay_n24 & 1283170 & 9576 & $9847(0 \%)$ \\
\hline road_usa & 622338 & 1065 & $1151(1 \%)$ \\
\hline
\end{tabular}

RMS WAVEFRONT METRICS FOR THE DIFFERENT ALGORITHMS. BEST RESULTS APPEAR IN BOLD.

collection of Fortran codes for large-scale scientific computations. In particular, we compare against the MC60 software package, which implements both serial RCM and Sloan. We did not find a reordering implementation of BFS. To measure how the various permutations affect performance and to present results for end-to-end execution times, we used the implementation of SpMV as found in PETSc library, version 3.4 [四].

To compute the source nodes for reordering, we use the algorithm by Kumfert [29] described in Section एᄑ-E. As previously mentioned, the main computation during this phase corresponds to repeated BFS traversals that we parallelized using the unordered BFS algorithm. Our parallel algorithms use the nodes computed with our parallel implementation of this algorithm, while HSL uses the nodes it finds with its own implementation of the same algorithm. We noticed that the nodes produced are usually different.

The experiments for reordering were ran on the Stampede supercomputer hosted at the University of Texas at Austin. Each node of the cluster runs CentOS Linux, version 6.3 and consists of 2 Xeon Processors of 8 cores each, operating at $2.7 \mathrm{GHz}$. Each core has a unified $256 \mathrm{~KB}$ L2 cache and each processor has a shared 20MB L3 cache. A node contains 32GB of main memory.

\begin{tabular}{lrrrrr}
\hline & \multicolumn{2}{c}{$\begin{array}{c}\text { PseudoDiameter } \\
\text { and Reordering }\end{array}$} & & \multicolumn{2}{c}{ Only Reordering } \\
\cline { 2 - 3 } Input & RCM & Sloan & & RCM & Sloan \\
\hline inline_1 & 0.41 & 0.71 & & 0.13 & 0.44 \\
audikw_1 & 1.11 & 1.82 & & 0.29 & 1.00 \\
dielFilterV3real & 1.03 & 1.84 & & 0.29 & 1.11 \\
G3_circuit & 0.41 & 0.81 & & 0.10 & 0.51 \\
M6 & 3.83 & 5.57 & & 0.68 & 2.42 \\
333SP & 1.98 & 3.39 & & 0.48 & 1.88 \\
NLR & 3.47 & 5.76 & & 0.83 & 3.11 \\
hugetric-00020 & 4.83 & 7.85 & & 1.06 & 4.07 \\
delaunay_n24 & 5.82 & 13.83 & & 1.79 & 9.80 \\
road_usa & 8.27 & 12.56 & & 2.15 & 6.43 \\
\hline
\end{tabular}

HSL EXECUTION TIMES IN SECONDS

\section{B. Reordering Quality}

Tables $\mathbf{W}$ and $\mathbf{W}$ show the bandwidth ${ }^{\square}$ and root mean square (RMS) of the wavefront, respectively. Since Sloan is designed to reduce wavefront (and not bandwidth), Table $\mathbb{l l}$ shows the bandwidth only for BFS and RCM. Similarly, Table UI shows RMS wavefront for Sloan but not for BFS or RCM. For each metric, Tables $\mathbf{~ W l}$ and $\mathbf{I I}$ show the initial matrix and the values after applying the permutation. Columns labeled HSL show the values of the corresponding metric for HSL implementations. In Table $\mathbb{W}$ we show only one value for RCM because both the leveled and unordered RCM programs produce the same value. In the case of BFS and Sloan, each parallel execution can produce a different permutation. Thus, for each matrix running with a number of threads between 2 and 16 (in steps of 2), we ran the appropriate program five times and report the mean value and the standard deviation as a percentage relative to its mean.

Table $\mathbb{l}$ shows that HSL's sequential RCM and our parallel RCM produce very similar bandwidth numbers. Some differences appear for delaunay_n24, hugetric-00020, and $333 S P$. However, these differences are negligible (less than $0.002 \%$ ) when compared with the initial bandwidth. The BFS reordering generally produces worse bandwidth, and standard deviation shows that the variation due to the non-deterministic results is usually not significant.

Table WU shows RMS wavefront for our Sloan implementation when running in parallel. Again, while parallel Sloan is better than HSL's sequential Sloan in three cases, the results of our parallel algorithm follow closely those of HSL in all cases. Also, in our experience, when run in parallel, Sloan's metrics do not differ much from the wavefront produced with just one thread, which suggests that the relaxations of the priority queue and atomicity of graph updates do not significantly affect the resulting quality of the reordering for these inputs.

\section{Reordering Performance}

In this section, we compare the execution times of different reordering algorithms. Table $\mathbb{D V}$ shows the execution times for HSL sequential algorithms. The first two columns correspond to the overall time spent by HSL's RCM and HSL's Sloan

\footnotetext{
${ }^{2}$ Since our matrices are symmetric, the bandwidth is the semi-bandwidth of the matrix.
} 

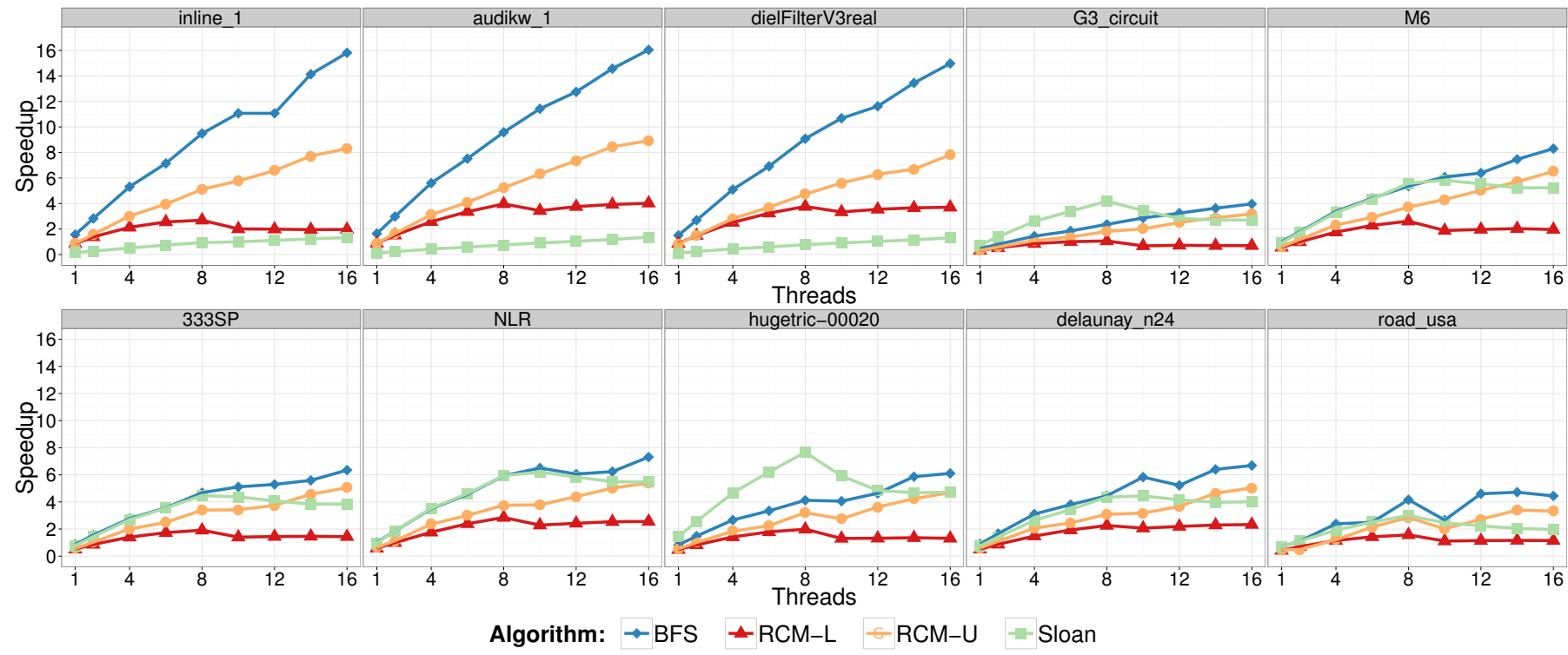

Fig. 2. Speedup scaling of reordering with the number of threads versus HSL. Pseudo-diameter computation is excluded from the parallel as well as the sequential versions.

\begin{tabular}{|c|c|c|c|c|c|c|c|c|c|c|c|c|c|c|c|c|}
\hline \multirow[b]{2}{*}{ Input } & \multicolumn{5}{|c|}{ Reorder Time } & \multicolumn{6}{|c|}{ SpMV Time } & \multicolumn{5}{|c|}{ Breakeven Iterations } \\
\hline & HSL-RCM & HSL-Sloan & BFS & $\mathrm{RCM}$ & Sloan & Natural & HSL-RCM & HSL-Sloan & BFS & $\mathrm{RCM}$ & Sloan & HSL-RCM & HSL-Sloan & BFS & $\mathrm{RCM}$ & Sloan \\
\hline inline_1 & 0.41 & 0.71 & 0.08 & 0.09 & 0.47 & 0.87 & 0.54 & 0.54 & 0.54 & 0.54 & 0.55 & 124 & 217 & 24 & 27 & 148 \\
\hline dielFilterV3real & 1.03 & 1.84 & 0.12 & 0.13 & 1.09 & 2.51 & 1.35 & 1.36 & 1.40 & 1.35 & 1.36 & 89 & 160 & 10 & 12 & 95 \\
\hline G3_circuit & 0.41 & 0.81 & 0.16 & 0.17 & 0.35 & 0.36 & 0.21 & 0.21 & 0.21 & 0.21 & 0.22 & 269 & 541 & 112 & 114 & 254 \\
\hline M6 & 3.83 & 5.57 & 0.79 & 0.74 & 1.08 & 2.81 & 0.53 & 0.58 & 0.55 & 0.53 & 0.57 & 169 & 250 & 35 & 33 & 49 \\
\hline 333SP & 1.98 & 3.39 & 0.39 & 0.42 & 0.90 & 2.66 & 0.59 & 0.62 & 0.65 & 0.62 & 0.62 & 96 & 166 & 19 & 20 & 44 \\
\hline hugetric-00020 & 4.83 & 7.85 & 1.62 & 1.68 & 2.01 & 2.84 & 0.78 & 0.89 & 0.79 & 0.76 & 0.92 & 235 & 402 & 79 & 81 & 105 \\
\hline delaunay_n24 & 5.82 & 13.83 & 1.66 & 1.82 & 3.89 & 7.51 & 2.71 & 3.66 & 2.77 & 2.73 & 3.63 & 121 & 359 & 35 & 38 & 100 \\
\hline road_usa & 8.27 & 12.56 & 3.91 & 4.32 & 6.92 & 5.52 & 2.43 & 2.97 & 2.56 & 2.43 & 3.09 & 267 & 491 & 132 & 140 & 284 \\
\hline
\end{tabular}

TABLE VI

TIME FOR REORDERING AND PERFORMING 100 ITERATIONS OF SPARSE MATRIX-VECTOR MULTIPLY WITH 16 THREADS. TIMES IN SECONDS. BREAKEVEN ITERATIONS IS THE NUMBER OF ITERATIONS FOR TIME WITH REORDERING TO SURPASS NO REORDERING (I.E., NATURAL).

\begin{tabular}{|c|c|c|c|c|c|c|c|c|}
\hline \multirow[b]{3}{*}{ Input } & \multicolumn{2}{|c|}{ BFS } & \multicolumn{4}{|c|}{ RCM } & \multicolumn{2}{|c|}{ Sloan } \\
\hline & \multirow[b]{2}{*}{ Speedup } & \multirow[b]{2}{*}{$\begin{array}{l}\text { Relative } \\
\text { Quality }\end{array}$} & \multicolumn{2}{|c|}{ Leveled } & \multicolumn{2}{|c|}{ Unordered } & \multirow[b]{2}{*}{ Speedup } & \multirow[b]{2}{*}{$\begin{array}{l}\text { Relative } \\
\text { Quality }\end{array}$} \\
\hline & & & Speedup & $\begin{array}{c}\text { Relative } \\
\text { Quality }\end{array}$ & Speedup & $\begin{array}{c}\text { Relative } \\
\text { Quality }\end{array}$ & & \\
\hline inline_1 & 5.07 & 0.89 & 2.71 & -0.00 & 4.63 & -0.00 & 1.52 & -0.01 \\
\hline audikw_1 & 6.42 & 0.76 & 4.25 & -0.03 & 6.14 & -0.04 & 1.77 & 0.08 \\
\hline dielFilterV3real & 18.85 & 0.91 & 5.07 & -0.00 & 7.67 & -0.00 & 1.69 & 0.07 \\
\hline G3_circuit & 2.48 & 0.99 & 1.19 & 0.00 & 2.38 & 0.00 & 2.31 & 0.04 \\
\hline M6 & 4.86 & 0.97 & 3.27 & -0.00 & 5.14 & -0.00 & 5.13 & 0.03 \\
\hline $333 \mathrm{SP}$ & 5.10 & 1.03 & 2.46 & 0.02 & 4.76 & 0.02 & 3.78 & -0.42 \\
\hline NLR & 3.05 & 0.98 & 2.14 & -0.00 & 2.92 & -0.00 & 3.66 & -0.01 \\
\hline hugetric-00020 & 2.98 & 0.91 & 1.93 & -0.03 & 2.88 & -0.03 & 3.91 & 0.03 \\
\hline delaunay_n24 & 3.50 & 1.42 & 1.97 & 0.22 & 3.21 & 0.22 & 3.56 & 0.03 \\
\hline road_usa & 2.11 & 0.95 & 1.31 & 0.00 & 1.91 & 0.00 & 1.81 & 0.09 \\
\hline Average & 4.12 & 0.97 & 2.45 & 0.01 & 3.89 & 0.01 & 2.74 & -0.02 \\
\hline
\end{tabular}

OVERALL SPEEDUP USING 16 THREADS VERSUS HSL OF PSEUDODIAMETER AND REORDERING COMPUTATIONS AND RELATIVE QUALITY.

to select source nodes and perform reordering, while the two columns under the label "only reordering" show how much time is spent in each HSL program excluding the pseudo- diameter computation.

Using these times as baselines, we present in Table $\nabla$ the parallel speedups when 16 cores are used, and in Figure \, we show how the speedups scale as the number of processors increases. For BFS, leveled RCM and unordered RCM, speedup is shown with respect to HSL's sequential RCM and for Sloan with respect to HSL's sequential Sloan. In Figure Z, we present the speedups after isolating the reordering computation from the selection of source nodes (compare with Table $\nabla$ which shows the overall time needed to compute the pseudo-diameter and perform reordering). Figure $\square$ shows that dietFilterV3real, audikw_l, and inline_l are the matrices with the best speedups for BFS and RCM. These matrices have a large number of nonzeros per row (approximately an average of 150 nonzeros per row). The other matrices have a lower number of nonzeros per row (less than 12 in all cases). Thus, speedups of parallel BFS-like traversals are higher for graphs with a larger number of edges per node.

Relative quality in Table $\nabla$ is the relative change of RMS 
wavefront for Sloan or bandwidth for the other algorithms compared to HSL. Positive values indicate worse quality relative to the HSL baseline. Our overall results in Table $\nabla$ show that both our leveled and unordered RCM algorithms achieve speedup over HSL implementations while still maintaining similar quality. The leveled algorithm achieves a geometric mean speedup of $2.45 \mathrm{X}$, and the unordered algorithm achieves a geometric average speedup of $3.89 \mathrm{X}$ when using 16 threads. In both cases, the geometric mean quality difference is very small, $1 \%$, and for some inputs, our implementations slightly improve the maximum bandwidth relative to HSL (i.e., relative quality less than zero). Additionally, the performance of our unordered RCM algorithm approaches the performance of $\mathrm{BFS}$, which is a reasonable upper bound on the performance of any graph traversal-based reordering algorithm.

In our past experience, unordered BFS performs better than ordered BFS for breadth-first traversals with a large number of levels. In an ordered implementation, a barrier must be placed between each level, while in the unordered implementation, barriers are avoided. The result of the pseudodiameter computation is to return two nodes that have a large distance between them, which means that the subsequent BFS will have many levels. Thus, one reason for the better performance of unordered RCM is that the heuristics used in RCM naturally lead to BFS with a large number of levels, which favors unordered traversal over ordered ones.

The parallel Sloan algorithm is also faster than the corresponding HSL implementation. We see an average speedup of 2.74X, while the quality remains similar.

A significant amount of time is spent computing the pseudodiameter nodes, which we have parallelized, but is not the subject of this paper. The "only reordering" column in Table $\mathbb{E}$ shows how much time is spent in each HSL program excluding the computation of these nodes, and Figure $\square$ shows the speedup of the different algorithms when only the execution time of the reordering algorithm itself is considered.

On average, the speedup of BFS, unordered RCM and Sloan, just considering the reordering time, is better than the speedup over the total computation, indicating that the pseudo-diameter computation is the scalability bottleneck. The geometric mean speedups on 16 cores for all matrices without the pseudo-diameter computation are 8.09X, 5.56X and 2.88X respectively. The speedups with the pseudo-diameter computation are $4.12 \mathrm{X}, 3.89 \mathrm{X}$ and $2.74 \mathrm{X}$ respectively. For leveled $\mathrm{RCM}$, the situation is reversed. The mean speedup is $1.95 \mathrm{X}$ without and $2.45 \mathrm{X}$ with the pseudo-diameter computation.

\section{End-to-end Performance}

The ultimate goal of reordering is to improve the performance of subsequent matrix operations. Thus, we consider the impact of our parallel reordering algorithm on sparse matrixvector product (SpMV), the key kernel in iterative methods.

To assess the impact that reordering has on SpMV, we evaluate a scenario where a user has a distributed matrix and reorders it locally to improve cache locality [2, 20]. Therefore, in this section, we show how local reordering will affect the

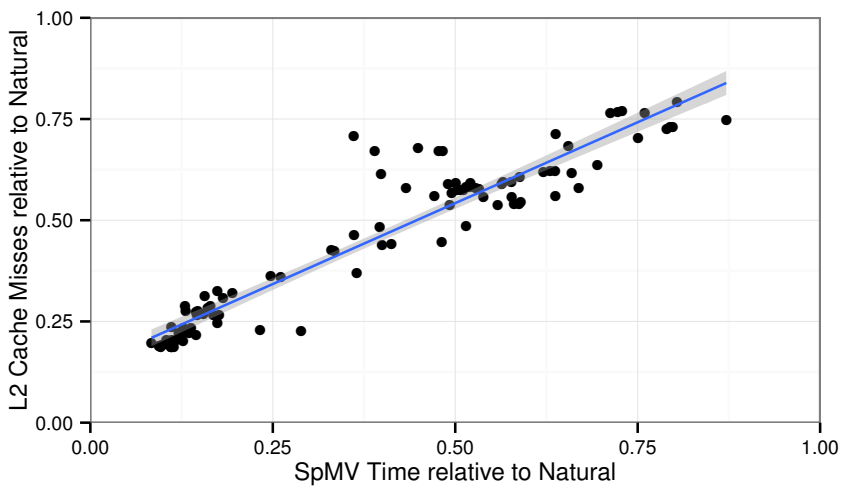

Fig. 3. Relationship between the number of caches misses in L2 and the benefit in SpMV running time. Cache misses and SpMV times for the reorderings are presented in relation to the ones obtained with natural ordering.

local part of the SpMV computation. For the experiments in this section, we use the same matrices that we used in the previous sections. In practice, each node will contain a slice of a large distributed matrix. However, it is reasonable to consider these matrices to be representative of those slices due to their size.

Table VI shows the times to run a hundred (100) SpMV iterations with matrices obtained by the different reorderings, using the implementation in the PETSc library and utilizing the 16 cores of a cluster node ${ }^{\text {ta }}$. For RCM, the table has a single column for both leveled and unordered RCM because both parallel RCM algorithms produce identical reorderings. Natural corresponds to the SpMV running times using the original matrix ordering. Using our parallel RCM reordering, the time to perform $100 \mathrm{SpMV}$ iterations is reduced by $1.5 \mathrm{X}$ compared to performing the SpMV iterations without reordering, and it is reduced by $2 \mathrm{X}$ compared to using HSL $\mathrm{RCM}$ for reordering.

Our experimental results show that RCM and BFS significantly decrease the execution time of SpMV. Since these reordering algorithms reduce the bandwidth of the matrix, they increase temporal and spatial locality in the accesses to the input vector since the vector elements accessed by operations on successive rows of the sparse matrix will have a large degree of overlap. To quantify this improvement in cache reuse, we measured the number of cache misses for SpMV using the matrices obtained with the different reorderings. Figure 3 shows the impact that the ratio in cache misses has on the ratio of SpMV running times when reordering is used in comparison with natural ordering. In Figure B] we present the ratios for L2 only since L2 cache misses show the strongest correlation between the reduction in cache misses and the reduction in SpMV running time. However, the data for L1 and L3 caches follow a similar pattern.

The experimental results also show that the execution times of SpMV kernels using the reorderings produced by our

\footnotetext{
${ }^{3}$ In a distributed iterative solver, SpMV iterations would be interleaved with inter-node communication.
} 
parallel implementations of RCM are similar to those using the HSL reordering. SpMV using the matrices reordered with $\mathrm{RCM}$ runs faster than those reordered with BFS, although in some cases the difference is small (see inline_1, G3_circuit and M6). Running times of the SpMV matrices reordered with Sloan are also significantly faster than those using the natural orderings and similar in most cases to those of HSL.

In this benchmark, the SpMV kernel is executed for one hundred (100) iterations. The actual number of SpMV iterations needed to solve a linear system approximately depends on many factors including the iterative algorithm that is used, the condition number of the matrix, and whether a good preconditioner is available. Table $\nabla]$ shows the break-even number of iterations that must be performed for reordering to pay off. This is computed taking into account the Reordering Times, also in Table VIl, that include the times to compute the pseudo-diameter, compute the reordering to produce a permutation vector, and permute the matrix and the result vector. For RCM, we show results only for the unordered $\mathrm{RCM}$. The reordering is done in parallel using all 16 cores, except for the HSL versions that runs serially.

Table $\nabla]$ shows that our parallel algorithms require less time for reordering than the corresponding serial HSL algorithms, and consequently need fewer SpMV iterations to amortize the reordering cost and run faster than Natural. Specifically, the parallel RCM and BFS require fewer SpMV iterations than sequential HSL-RCM, and the same is true for our parallel Sloan versus sequential HSL Sloan.

\section{RELATED WORK}

Following the publication of Cuthill-McKee algorithm for bandwidth reduction and subsequently Sloan algorithm for profile and wavefront reduction, most of the related work on this class of reordering algorithms has concentrated on improving the quality of the metrics [16, 18, 32, 34]. While most efforts follow a graph-based approach similar to RCM and Sloan, others are based on spectral reordering [5]. Several studies have shown that the best results are obtained when hybrid schemes that include RCM or Sloan as part of their computations are used [21, 25, 28, 35]. Still, all these previous approaches have only produced sequential implementations.

State-of-the-art sequential implementations of RCM and Sloan can be found in HSL software library [23] and Matlab [II]. HSL subroutines of RCM and Sloan are currently implemented using Fortran and their functionality is enhanced with features such as handling of supervariables. Alternative implementations of these algorithms can be found in the Boost Graph Library [42]. Implementations in Boost are simpler, but also less efficient when dealing with large matrices.

Little effort has gone into the parallelization of reordering algorithms. Previous works that address this problem are ParMetis [26] and PT-Scotch [10], which are both parallel versions of the nested dissection algorithm. Nested dissection reordering is used for graph partitioning and fill-in reduction, rather than bandwidth or wavefront reduction, and is considered a highly concurrent algorithm.
Several works discuss the parallelization of BFS algo-

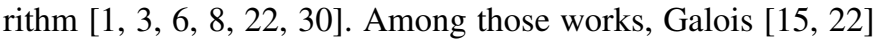
has implementations of the ordered and unordered implementations described here. Work by Beamer et al. [6] describes a new algorithm for BFS, which is geared towards scalefree graphs, which are not typical in numerical analysis. The rest implement variations of the ordered or level algorithm discussed in this paper. In any case, none of these works have evaluated BFS in the context of reordering.

\section{Conclusions}

Many sparse matrix computations benefit from reordering the sparse matrix before performing the computation. In this paper, we focused on one application of reordering: improving the cache locality of parallel iterative solvers. In these solvers, the key computation is a sparse matrix-vector product (SpMV), and reordering the matrix can improve the locality of accesses to the vector in the SpMV. In this application, reordering may take as much time as the solver, so parallel reordering algorithms are useful.

In this paper, we presented parallel implementations of two popular reordering algorithms, Reverse Cuthill-McKee (RCM) and Sloan. To our knowledge, these are the first such parallel implementations. Since both these reorderings are heuristics, we allow the parallel implementations to produce slightly different reorderings than those produced by the sequential implementations of these algorithms. Since this can affect the SpMV time, the impact on the overall time to solution has to determined experimentally.

In our experiments, we compared the quality of the reordered matrices obtained with these algorithms with those produced by the RCM and Sloan implementations in HSL mathematical software library. Our results show that our unordered RCM algorithm achieves speedups between 3.18X to $8.92 \mathrm{X}$ on 16 cores. These results match the performance of the more parallel BFS reordering algorithm on the same matrices, while producing similar quality results as sequential HSL-RCM. With our parallel RCM, fewer SpMV iterations are required to amortize the cost of reordering than are required for the state-of-the-art sequential HSL-RCM reordering. Reordering the matrix using our parallel RCM and then performing $100 \mathrm{SpMV}$ iterations is twice as fast as using HSL-RCM and then performing the SpMV iterations; it is also 1.5 times faster than performing the SpMV iterations without reordering the matrix.

These results suggest that the parallel reordering algorithms presented here should be part of the standard toolkit of HPC programmers.

\section{ACKNOWLEDGEMENTS}

The work presented in this paper has been supported by the National Science Foundation grants CNS 1111407, CNS 1406355, XPS 1337281, CCF 1337281, CCF 1218568, ACI 1216701, and CNS 1064956. We would also like to thank David Padua, Ahmed Sameh, Michael Heath and William 
Gropp for our fruitful discussions and their useful comments in the early stages of this work.

\section{REFERENCES}

[1] V. Agarwal, F. Petrini, D. Pasetto, and D. A. Bader. Scalable Graph Exploration on Multicore Processors. ACM/IEEE International Conference for High Performance Computing, Networking, Storage and Analysis (SC), 2010.

[2] W. K. Anderson, W. D. Gropp, D. E. K. Kaushik, and B. F. Smith Achieving High Sustained Performance in an Unstructured Mesh CFD Application. Technical report, Institute for Computer Applications in Science and Engineering (ICASE), 2000.

[3] D. A. Bader and K. Madduri. Designing Multithreaded Algorithms for Breadth-First Search and st-connectivity on the Cray MTA-2. International Conference on Parallel Processing (ICPP), 2006.

[4] S. Balay, J. Brown, K. Buschelman, W. D. Gropp, D. K. Kaushik, M. G. Knepley, L. C. McInnes, B. F. Smith, and H. Zhang. PETSc Web page, 2013. http://www.mcs.anl.gov/petsc.

[5] S. T. Barnard, A. Pothen, and H. D. Simon. A Spectral Algorithm for Envelope Reduction of Sparse Matrices. ACM/IEEE International Conference for High Performance Computing, Networking, Storage and Analysis (SC), 1993.

[6] S. Beamer, K. Asanović, and D. Patterson. Direction-Optimizing Breadth-First Search. ACM/IEEE International Conference for High Performance Computing, Networking, Storage and Analysis (SC), 2012.

[7] M. Benzi. Preconditioning Techniques for Large Linear Systems: A Survey. Journal of Computational Physics, 182(2), 2002.

[8] A. Buluç and K. Madduri. Parallel Breadth-First Search on Distributed Memory Systems. ACM/IEEE International Conference for High Performance Computing, Networking, Storage and Analysis (SC), 2011.

[9] D. Chazan and W. Miranker. Chaotic Relaxation. Linear Algebra and its Applications, 2(2), 1969.

[10] C. Chevalier and F. Pellegrini. PT-Scotch: A Tool for Efficient Parallel Graph Ordering. Parallel Computing, 34(6-8), 2008.

[11] CULA tools. http://www.culatools.com/blog/2011/12/19/2957/.

[12] E. Cuthill and J. McKee. Reducing the Bandwidth of Sparse Symmetric Matrices. 24th National Conference, 1969.

[13] T. A. Davis and Y. Hu. The University of Florida Sparse Matrix Collection. ACM Transactions on Mathematical Software (TOMS), $38(1), 2011$

[14] I. S. Duff, J. K. Reid, and J. A. Scott. The use of Profile Reduction Algorithms with a Frontal Code. International Journal for Numerical Methods in Engineering, 28, 1989.

[15] Galois System. http://iss.ices.utexas.edu/?p=projects/galois.

[16] A. George and J. W. H. Liu. Computer Solution of Large Sparse Positive Definite Systems. Prentice-Hall, 1981.

[17] O. Ghattas et al. CALIENTE Project. http://www.cs.cmu.edu/ caliente/.

[18] N. E. Gibbs, W. G. Poole, and P. K. Stockemeyer. An Algorithm for Reducing the Bandwidth and Profile of a Sparse Matrix. SIAM Journal on Numerical Analysis, 13(2), 1976.

[19] J. R. Gilbert, C. Moler, and R. Schreiber. Sparse Matrices in Matlab: Design and Implementation. SIAM Journal on Matrix Analysis and Applications, 13(1), 1992.

[20] W. D. Gropp, D. K. Kaushik, D. E. Keyes, and B. F. Smith. Performance Modeling and Tuning of an Unstructured Mesh CFD Application. ACM/IEEE International Conference for High Performance Computing, Networking, Storage and Analysis (SC), 2000.

[21] W. Hager. Minimizing the Profile of a Symmetric Matrix. SIAM Journal on Scientific Computing, 23(5), 2002.

[22] M. A. Hassaan, M. Burtscher, and K. Pingali. Ordered vs Unordered: A Comparison of Parallelism and Work-efficiency in Irregular Algorithms. ACM SIGPLAN Symposium on Principles and Practice of Parallel Programming (PPoPP), 2011.
[23] HSL (2011). A collection of Fortran Codes for Large Scale Scientific Computation. http://www.hsl.rl.ac.uk/.

[24] Y. Hu. Algorithms for Visualizing Large Networks. Combinatorial Scientific Computing. Chapman \& Hall, 2011.

[25] Y. F. Hu and J. A. Scott. A Multilevel Algorithm for Wavefront Reduction. SIAM Journal on Scientific Computing, 23(4), 2001.

[26] G. Karypis and V. Kumar. A Parallel Algorithm for Multilevel Graph Partitioning and Sparse Matrix Ordering. Journal of Parallel and Distributed Computing, 48, 1998.

[27] M. Kulkarni, K. Pingali, B. Walter, G. Ramanarayanan, K. Bala, and L. P. Chew. Optimistic Parallelism Requires Abstractions. ACM SIGPLAN Conference on Programming Language Design and Implementation (PLDI), 2007.

[28] G. Kumfert and A. Pothen. Two Improved Algorithms for Envelope and Wavefront Reduction. BIT Numerical Mathematics, 37(3), 1997.

[29] G. K. Kumfert. An Object-Oriented Algorithmic Laboratory For Ordering Sparse Matrices. PhD thesis, Old Dominion Unversity, 2000.

[30] C. E. Leiserson and T. B. Schardl. A Work-Efficient Parallel BreadthFirst Search Algorithm (or How to Cope with the Nondeterminism of Reducers). ACM Symposium on Parallelism in Algorithms and Architectures (SPAA), 2010.

[31] A. Lenharth, D. Nguyen, and K. Pingali. Priority Queues Are Not Good Concurrent Priority Schedulers. Technical Report TR-11-39, University of Texas at Austin, 2012.

[32] J. G. Lewis. Implementation of the Gibbs-Poole-Stockmeyer and Gibbs-King Algorithms. ACM Transactions on Mathematical Software (TOMS), 8(2), 1982.

[33] R. Lipton, D. Rose, and R. Tarjan. Generalized Nested Dissection. SIAM Journal on Numerical Analysis, 16(2), 1979.

[34] W.-H. Liu and A. H. Sherman. Comparative Analysis of the CuthillMcKee and the Reverse Cuthill-McKee Ordering Algorithms for Sparse Matrices. SIAM Journal on Numerical Analysis, 13(2), 1976.

[35] M. Manguoglu, M. Koyutürk, A. H. Sameh, and A. Grama. Weighted Matrix Ordering and Parallel Banded Preconditioners for Iterative Linear System Solvers. SIAM Journal on Scientific Computing, 32(3), 2010.

[36] C. Mueller, B. Martin, and A. Lumsdaine. A Comparison of Vertex Ordering Algorithms for Large Graph Visualization. International AsiaPacific Symposium on Visualization (APVIS), 2007.

[37] D. Padua. Multiprocessors: Discussion of some Theoretical and Practical Problems. PhD thesis, University of Illinois, Urbana-Champaign, 1979.

[38] C. Papadimitriou. The NP-Completeness of the Bandwidth Minimization Problem. Computing, 16(3), 1976.

[39] J. C. Pichel, F. F. Rivera, M. Fernández, and A. Rodríguez. Optimization of Sparse Matrix-Vector Multiplication using Reordering Techniques on GPUs. Microprocessors \& Microsystems, 36(2), 2012.

[40] K. Pingali and et al. The Tao of Parallelism in Algorithms. ACM SIGPLAN Conference on Programming Language Design and Implementation (PLDI), 2011.

[41] A. J. K. Reid and A. J. A. Scott. Ordering Symmetric Sparse Matrices for Small Profile and Wavefront. Technical Report RAL-1998-016, Rutherford Appleton Laboratory, 1998.

[42] J. G. Siek, L.-Q. Lee, and A. Lumsdaine. The BOOST Graph Library: User Guide and Reference Manual. Addison-Wesley, 2002.

[43] S. Sloan. An Algorithm for Profile and Wavefront Reduction of Sparse Matrices. International Journal for Numerical Methods in Engineering, 23(2), 1986

[44] S. Sloan. A Fortran Program for Profile and Wavefront Reduction. International Journal for Numerical Methods in Engineering, 28, 1989. 